\title{
On the Dynamic Flows in Networks
}

\author{
Erusalimskiy Iakov
}

\begin{abstract}
Institute of Mathematics, Mechanics and Computer Sciences, Sothern Federal University, Rostov-on- Don, 344006, Bolshaya Sadovaya 105, Russia

*Corresponding Author: ymerusalimskiy@sfedu.ru; dnjme@math.sfedu.ru
\end{abstract}

Copyright (C) 2014 Horizon Research Publishing All rights reserved.

\begin{abstract}
Considered dynamic network flows identically zero on the negative time axis. The concept of the frontal flow is introduced for such flow. The article contains the theorem about the decomposition of the dynamic flow on an arbitrary time interval $[0 ; T]$ on the sum of two flows, the first of which is equivalent to the original flow on the interval and equals to zero outside the interval, the second flow is equivalent to zero on the interval and coincides with the initial flow outside of the interval. The first is minimum flow which equals the original flow at a given time interval.
\end{abstract}

Keywords Network, Dynamic Flow, Partial Flow, Frontal Flow

\section{Introduction}

The subject in the article is the dynamic flows in oriented networks which identically equals to zero for $t<0$. We define the concepts of the part of the flow and the front flow for such flow Decomposition theorem for dynamic flow on the time interval $[0 ; T]$ in the sum of frontal flows are proved. The main result is decomposition theorem for the dynamic flow which identically equals to zero for $t<0$ as the sum of two flows. The first flow has the same magnitude as original flow on $[0 ; T]$, and it is identically equal to zero for $t>T$, the second flow is equal to original flow for $t>T$, and it is magnitude equals to zero on $[0 ; T]$.

\section{Definitions and Notations}

Let $G(X, U, f, c)$ is a oriented network, where $X$ a set of vertices; $U$ - set of arcs; $f: U \rightarrow X \times X$. Denote $s$-a source, $r$-a drain. The capacity of each arc we denote $c(u), U_{-}(x)$ - the set of arcs entering to the vertex $x$; $U_{+}(x)$ - the set of arcs exiting out the vertex $x$.

Now we recall the definition of a stationary flow in the network (see [1]).

\section{Definition 1: Stationary flow}

Stationary flow in the network $G(X, U, f, c)$ is a mapping $\varphi: U \rightarrow R_{+}=(o ; \infty)$, which satisfying the following conditions:

$$
\left\{\begin{array}{l}
\sum_{u \in U_{-}(x)} \varphi(u)=\sum_{u \in U_{+}(x)} \varphi(u) ; x \in X, x \neq s, x \neq r . \\
0 \leq \varphi(u) \leq c(u), u \in U
\end{array}\right.
$$

where $s$ - the source; $r$ - the drain.

Magnitude of a stationary flow is called flow values sum through any arcs belongs some cut of the network, and in particular,

$$
\sum_{u \in U_{-}(r)} \varphi(u)=\sum_{u \in U_{+}(s)} \varphi(u)
$$

When we talk about the dynamic flow (see [2 - 5]), the discrete time $t \in Z$ is added to consideration. It is believed that each transition along the arc is made during the unit of discrete time (i.e., per clock).

Here is the definition of dynamic flow:

\section{Definition 2: Dynamic flow}

Dynamic flow in the network $G(X, U, f, c)$ is a mapping $\varphi: U \times Z \rightarrow R_{+}$, for a which the next conditions satisfy:

$$
\left\{\begin{array}{l}
\sum_{u \in U_{-}(x)} \varphi(u, t)=\sum_{u \in U_{+}(x)} \varphi(u, t+1) ; x \in X, x \neq s, x \neq r, t \in Z \\
0 \leq \varphi(u, t) \leq c(u), u \in U, t \in Z .
\end{array}\right.
$$

where $s$ - the source; $r$ - the drain; $t$ - the time.

\section{Definition 3: Instantaneous magnitude of the flow}

The magnitude of the flow $\varphi$ at the time $t$ is a value $v(\varphi, t)$ determined by the equals :

$$
v(\varphi, t)=\sum_{u \in U_{-}(r)} \varphi(u, t) .
$$


I.e. the total flow entering the drain at the time $t$.

\section{Definition 4: Magnitude of the flow on interval}

The magnitude of the flow $\varphi$ on the interval $\left[T_{1} ; T_{2}\right]_{Z}$ (here in after instead $\left[T_{1} ; T_{2}\right]_{Z}$ we write $\left.\left[T_{1} ; T_{2}\right]\right)$ is the value $V\left(\varphi,\left[T_{1}, T_{2}\right]\right)$ defined by:

$$
V\left(\varphi,\left[T_{1} ; T_{2}\right]=\sum_{t=T_{1}}^{T_{2}} \sum_{u \in U_{-}(r)} \varphi(u, t)\right.
$$

I.e. the total flow coming into the drain during the time interval $\left[T_{1} ; T_{2}\right]$

\section{Claims}

It is clear that we have the equality

$$
\begin{aligned}
& \sum_{u \in U} \varphi(u, t)=\sum_{u \in U_{+}(s)} \varphi(u, t)+\sum_{u \in U \backslash\left(U_{+}(s) \cup U_{-}(r)\right)} \varphi(u, t)+ \\
& +\sum_{u \in U_{-}(r)} \varphi(u, t)= \\
& =\sum_{u \in U_{+}(s)} \varphi(u, t)+\sum_{u \in U \backslash\left(U_{+}(s) \cup U_{-}(r)\right)} \varphi(u, t)+V(\varphi, t)
\end{aligned}
$$

Dynamic flow definition implies:

$$
\begin{aligned}
& \sum_{u \in U_{+}(s)} \varphi(u, t)+\sum_{u \in U \backslash\left(U_{+}(s) \cup U_{-}(r)\right)} \varphi(u, t) \\
= & \sum_{u \in U \backslash\left(U_{+}(s) \cup U_{-}(r)\right)} \varphi(u, t+1)+\sum_{u \in U_{-}(r)} \varphi(u, t+1) \\
= & \sum_{u \in U \backslash\left(U_{+}(s) \cup U_{-}(r)\right)} \varphi(u, t+1)+V(\varphi, t+1) .
\end{aligned}
$$

Denote

$$
\begin{aligned}
& I(t)=\sum_{u \in U_{+}(s)} \varphi(u, t), I I(t)=\sum_{u \in U_{+}(s)} \varphi(u, t), \\
& I I I(t)=\sum_{u \in U_{-}(r)} \varphi(u, t)
\end{aligned}
$$

We write down the equality that follows from (5) and (6):

$$
I(t)+I I(t)+I I I(t)=I I(t+1)+I I I(t+1)+I I I(t) .
$$

Consider the flow is identically equal to zero for $t<0$, and let $T>0, T \in Z$.

For this flow we have (for $t=0,1, \ldots, T-1$ ) next equalities:

$$
\begin{aligned}
& I(0)+I I(0)+I I I(0)=I I(1)+I I I(1)+I I I(0) \\
& I(1)+I I(1)+I I I(1)=I I(2)+I I I(2)+I I I(1)
\end{aligned}
$$

$$
\begin{gathered}
I(2)+I I(2)+I I I(2)=I I(3)+I I I(3)+I I I(2) \\
I(T-1)+I I(T-1)+I I I(T-1)=I I(T)+I I I(T)+I I I(T-1)
\end{gathered}
$$

Add to these equalities the following identity

$$
I(T)+I I(T)+I I I(T)=I(T)+I I(T)+I I I(T) .
$$

Sum the resulting set of equalities:

$\sum_{t \in[0 ; T]} I(t)+I I(0)+I I I(0)=\sum_{t \in[0 ; T-1]} I I I(t)+I(T)+I I(T)+I I I(T)$

From the fact that the flow $\varphi$ is identically equal to zero for $t<0$ the next equalities satisfied $I I(0)=0, \quad I I I(0)=0$. We have obtained the equality:

$$
\begin{aligned}
& \sum_{t \in[0 ; T]} I(t)=\sum_{t \in[0 ; T-1]} I I I(t)+I(T)+I I(T)+I I I(T) \\
& =V(\varphi,[0 ; T-1])+I(T)+I I(T)+I I I(T) .
\end{aligned}
$$

The expression on the left-hand side of equality (8) can be called the total flow entering the network at an interval $[0 ; T]$. Thus we have proved

Theorem 1 For any dynamic flow $\varphi$ is identically equal to zero for $t<0$ and for all $T>0, T \in Z$ there is a basic balance relation:

$$
\sum_{t \in[0 ; T]} I(t)=V(\varphi,[0 ; T-1])+I(T)+I I(T)+I I I(T) .
$$

It is obvious that for any dynamic flow $\varphi$ and arbitrary $T_{1}<T_{2}, T_{1}, T_{2} \in Z$ we have the following balance relation:

$$
\begin{aligned}
& I\left(T_{1}\right)+I I\left(T_{1}\right)+I I I\left(T_{1}\right)+\sum_{t \in\left[T_{1}+1 ; T_{2}\right]} I(t) \\
& =V\left(\varphi,\left[0 ; T_{2}-1\right]\right)+I\left(T_{2}\right)+I I\left(T_{2}\right)+I I I\left(T_{2}\right) .
\end{aligned}
$$

\section{Definition 5: Partial flow}

Let $G(X, U, f, c)$ is oriented network, ${ }^{\varphi}$ is dynamic flow. Dynamic flow $\varphi_{1}$ will be called the partial flow for $\varphi$, if all time ${ }^{t}$, and any arc $u$ satisfied:

$$
0 \leq \varphi_{1}(u, t) \leq \varphi(u, t) .
$$

Obviously, there are properties:

a) Zero flow is the partial flow of any flow.

b) Any flow is the partial flow of itself.

c) If the flow $\varphi_{1}$ is the partial flow of the flow $\varphi$ and the flow $\varphi_{2}$ is the partial flow of the flow $\varphi_{1}$, then flow $\varphi_{2}$ is the partial flow of the flow $\varphi$.

d) If the flow $\varphi_{1}$ is the partial flow of the flow $\varphi$, then the flow $\varphi-\varphi_{1}$ is also the partial flow of the flow $\varphi$.

We continue to study flows are identically zero for $t<0$. 


\section{Definition 6: Equivalent flows}

Two dynamic flow $\varphi_{1}$ and $\varphi_{2}$ will be called equivalent on the time interval $\left[T_{1} ; T_{2}\right]_{Z}, T_{1} \leq T_{2}, T_{1}, T_{2} \in Z$ if for any $t \in\left[T_{1} ; T_{2}\right]_{Z}$ next equality $V\left(\varphi_{1}, t\right)=V\left(\varphi_{2}, t\right)$ holds.

Example 1. Consider the network shown in Fig. 1. We assume that the capacity of all arcs is equals 2 . Consider two flows $\varphi_{1}$ and $\varphi_{2}$ shown in Fig. 2 and Fig. 3, respectively, which will be assumed to be constant at the time. Figures standing near arcs indicate the value of the flow.

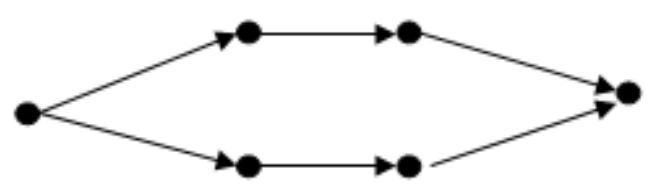

Fig. 1.

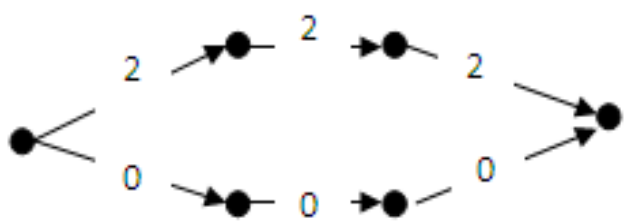

Fig. 2.

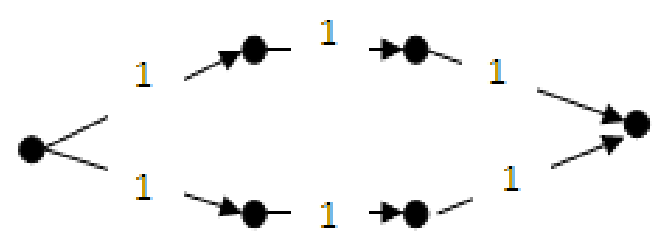

Fig. 3.

It is clear that the flows in Fig. 2 and Fig. 3 equivalent at any time interval $\left[T_{1} ; T_{2}\right]_{Z}, T_{1} \leq T_{2}, T_{1}, T_{2} \in Z$, including at all $Z$.

It is clear that any flow is equivalent to itself at all $Z$.

\section{Definition 7: Frontal flow}

Let $G(X, U, f, c)$ is oriented network, $\varphi$ - dynamic flow identically equal to zero for $t<0$. Frontal flow of the flow $\varphi$ will be called a flow $\varphi_{F}$ satisfied the following conditions:

a) $\varphi_{F}$ is a the partial flow of the flow $\varphi$; b) $\varphi_{F}$ is a the partial flow of the flow $\varphi$;

c) $\varphi_{F}(u, 0)=\varphi(u, 0), \forall u \in U$;

d) $\varphi_{F}(u, t)=0, \forall u \in U_{+}(s), \forall t \geq 1$.

Theorem $2 \Delta$ For any dynamic flow $\varphi$ that is identically equal to zero when $t<0$ there is frontal flow $\varphi_{F}$.

Proof: Frontal flow $\varphi_{F}$ is the partial flow of the flow $\varphi$. From (11) implies that it is identically equal to zero for $t<0$. Its value when $t=0$ is determined under definition 6 (c). Next we argue by induction. Let us assume that the frontal flow is already defined for all $t \leq t_{0}$. We show that it is defined and when $t=t_{0}+1$.

Take $w$ - arbitrary arc of the network $G(X, U, f, c)$. If $w \in U_{+}(s)$, then $\varphi_{F}\left(u, t_{0}+1\right)=0$ (see definition 7 (c)). If $w \in U \backslash U_{+}(s)$, then we find a vertex $x_{w}$ that $u \in U_{+}\left(x_{w}\right)$.

Let

$$
\sum_{u \in U_{-}\left(x_{w}\right)} \varphi\left(u, t_{0}\right) \stackrel{\text { def }}{=} b_{w} \text { and } \sum_{u \in U_{-}\left(x_{w}\right)} \varphi_{F}\left(u, t_{0}\right) \stackrel{\text { def }}{=} a_{w}
$$

It is clear that

$$
a_{w} \leq b_{w}
$$

This follows from partial flow definition

If $\left(a_{u}=0\right) \vee\left(b_{u}=0\right)$, then we believe $\varphi_{F}\left(u, t_{0}+1\right)=0$. (This follows from the non-negativity of the flow.) It remains to consider the case $0<a_{w} \leq b_{w}$. If $\varphi\left(w, t_{0}+1\right)=0$ we believe $\varphi_{F}\left(w, t_{0}+1\right)=0$. (It requires partial flow definition.) Otherwise, in the set $U_{+}\left(x_{w}\right)$ exists a non-empty subset of the set of $\operatorname{arcs} U_{+\varphi}\left(x_{w}\right)$, such that:

a) $\sum_{u \in U_{+\varphi}\left(x_{w}\right)} \varphi\left(u, t_{0}+1\right)=b_{w}$;

b) $\varphi\left(u, t_{0}+1\right)>0, \forall u \in U_{+\varphi}\left(x_{w}\right)$;

c) $w \in U_{+\varphi}\left(x_{w}\right)$.

We numerate arcs of the set $U_{+\varphi}\left(x_{w}\right)=\left\{u_{1}, u_{2}, \ldots u_{k}\right\}$. Let $w=u_{1}$.

It is clear that

$$
\sum_{i=1}^{k} \varphi\left(u_{i}, t_{0}+1\right)=b_{w}
$$

Consider the equation:

$$
\sum_{i=1}^{k} \varphi\left(u_{i}, t_{0}+1\right)=b_{w} .
$$

From (13) and (14) it follows that the equation (15) has solutions. (In the case $a_{w}=b_{w}$ this solution is unique.) Fix 
a solution of equation (15) - $\left(\widetilde{x}_{1}, \widetilde{x}_{2}, \ldots, \widetilde{x}_{k}\right)$. We believe in this case

$$
\begin{aligned}
& \varphi_{F}\left(w, t_{0}+1\right)=\tilde{x}_{1}, \\
& \varphi_{F}\left(u_{2}, t_{0}+1\right)=\tilde{x}_{2}, \ldots, \varphi_{F}\left(u_{k}, t_{0}+1\right)=\tilde{x}_{k}
\end{aligned}
$$

Note that in this way we have defined $\varphi_{F}$ on all arcs of the set $U_{+\varphi}\left(x_{w}\right)=\left\{u_{1}, u_{2}, \ldots u_{k}\right\}$ within $t=t_{0}+1$. The inductive step is proved.

Now we consider the flow $\varphi_{1}=\varphi-\varphi_{F}$. It is clear that it is identically equal to zero for $t<1$.

Similarly, the definition 7, we can determine its frontal flow (replacement $t=0$ for $t=1)-\varphi_{1 F}$. Consider the flow $\varphi_{2}=\varphi_{1}-\varphi_{1 F}=\varphi-\varphi_{F}-\varphi_{1 F}$. It is clear that it is identically equal to zero for $t<2$. Thus, the following theorem holds.

Theorem 3 For any dynamic flow $\varphi$ on oriented network that is identically equal to zero for $t<0$, and $T>0, T \in Z$ equality holds:

$$
\begin{aligned}
& \varphi(u, t)=\varphi_{F}(u, t)+\varphi_{1 F}(u, t) \\
& +\cdots+\varphi_{T F}(u, t), \forall t \leq T
\end{aligned}
$$

Equation (16) we call the decomposition of the flow across on $[0 ; T]$ in the sum of frontal flows.

\section{Definition 8: The conveying path}

Let $G(X, U, f, c)$ - oriented network with a source $s$ and a drain $r . \mu:[1 ; m]_{N} \rightarrow U$ - the path from $s$ to $r$. They say that the path $\mu$ carries a portion $\Delta$ of the $\left[T_{1} ; T_{2}\right]$, if there is a dynamic flow $\varphi_{\mu}$ that satisfies the following conditions:

a) $\varphi_{\mu}$ is identically zero outside the interval $\left[T_{1} ; T_{2}\right]$;

b) $\varphi_{\mu}\left(\mu\left(t-T_{1}+1\right), t\right)=\Delta$,

$$
\varphi_{\mu}(u, t)=0, u \neq \mu\left(t-T_{1}+1\right) \text {. }
$$

It is clear that for conveying path next equalities satisfies

$$
v\left(\varphi_{\mu}, T_{2}\right)=\Delta, \quad v\left(\varphi_{\mu}, t\right)=0, t \neq T_{2}, V\left(\varphi_{\mu},\left[T_{1} ; T_{2}\right]\right)=\Delta .
$$

Theorem 4 Theorem 4 Let $\varphi(u, t)$ a dynamic flow on network $G(X, U, f, c)$ equal to zero on all arcs of the network when $t<0$, then for an $\operatorname{arbitrary} T \in N$, there are flows $\varphi_{1}(u, t), \varphi_{2}(u, t)$ for which the following conditions are satisfy:

a) Flows $\varphi_{1}(u, t), \quad \varphi_{2}(u, t)$ - partial flows for the flow $\varphi(u, t)$;

b) Flow $\varphi_{1}(u, t)$ is equal to zero on all arcs with $t>T$;

c) Flow $\varphi_{1}$ is equivalent to the flow $\varphi$ on interval

$$
[0 ; T] \text {, i.e. } V(\varphi, t)=V\left(\varphi_{1}, t\right), t \in[0 ; T] ;
$$

d) Flow $\varphi_{2}$ is equivalent to zero on interval $[0 ; T]$, i.e. $V\left(\varphi_{2}, t\right)=0, t \in[0 ; T]_{Z}$;

e) $\varphi(u, t)=\varphi_{1}(u, t)+\varphi_{2}(u, t), \quad t \in Z$.

Note that the flow $\varphi_{2}$ is equal to the flow ${ }^{\varphi} 1$ for all $t>T$.

Proof: $\Delta$ In fact, it suffices to prove this theorem for the frontal flow.

Consider frontal flow $\varphi_{i F}, i \in[0 ; T], \varphi_{0 F} \stackrel{\text { def }}{=} \varphi_{F}$. It is clear that

$v\left(\varphi_{i F}, t\right)=0$ when $t<i$;

If $v\left(\varphi_{i F}, t\right)>0$, then $t \in[i ; T]$ and the values $\varphi_{i f}(u, t)$ obtained on the arcs of the set $U_{-}(r)$ of values taken on the set of $\operatorname{arcs} U_{+}(s)$ in time $t$ shipment of paths which length equal $t-i+1$.

The network is a finite graph and we can solve the problem of transportation quantity $v\left(\varphi_{i F}, t\right)$ with the paths have a given length. These paths are parts of a flow $\varphi_{i F}$. Define transporting paths for all $t \in[i ; T]$. The desired expansion has the form

$$
\begin{gathered}
\left(\varphi_{i f}(u, t)\right)_{1}=\text { "The sum of transporting flows" } \\
\left(\varphi_{i f}(u, t)\right)_{2}=\varphi_{i f}(u, t)-\left(\varphi_{i f}(u, t)\right)_{1} \nabla
\end{gathered}
$$

Decomposition of the frontal flow in sum of of conveying flows is an analogue of the theorem on the decomposition of a stationary flow in sum of flow along paths (see [6]).

From basic balance relation (9) we have that the partial flow $\varphi_{1}(u, t)$ of the flow $\varphi(u, t)$ (obtained by theorem 3 ) it is minimum partial flow satisfying

$$
V(\varphi,[0 ; T])=V\left(\varphi_{1},[0 ; T]\right),
$$

i.e. $\varphi_{1}$ is minimum flow which equals the original flow at a given time interval.

\section{REFERENCES}

[1] L. R. Ford, Jr. \& D. R. Fulkerson Flows in Networks (With a new foreword by Robert G. Bland and James B. Orlin)//Princeton University Press, 2010. 216 Pp.

[2] Aronson J.E. A survey of dynamic network flows. / J.E. Aronson // Annals of Operations Research. -No. 20 -1989. Pp.1-66.

[3] L.R. Ford. Constructing Maximal Dynamic Flows from Static Flows. / L.R. Ford, D.R. Fulkerson // Operations Research. -1958 -Vol.6 - Pp.419-433.

[4] Skutella M. An Introduction to Network Flows Over Time. / 
M. Skutella // Research Trends in Combinatorial Optimization. Berlin: Springer Berlin Heidelberg, -2009. pp.451-482. Sleator D.D. A data structure for dynamic trees. / D.D. Sleator, R.E. Tarjan. // J. Comput. Syst. Sci. -1983 . -No.26. - pp.362-391.

[5] Erusalimskiy Y.M. Unsteady flow in a network / Y.M.
Erusalimskiy, N.N. Vodolazov // Vestnik DGTU. -2009. -Vol. 9, \#3 (42) - Pp.402-409.

[6] Busaker Robert G., Saaty Thomas L. Finite Graphs and Networks//Mc. Graw-Hill Book Company, New York, 1965, $294 \mathrm{Pp}$. 